\title{
Macroscopic Dark Matter Constraints from Bolide Camera Networks
}

\author{
Jagjit Singh Sidhu, Glenn Starkman \\ Physics Department/CERCA/ISO Case Western Reserve University Cleveland, Ohio 44106-7079, USA
}

(Dated: December 13, 2019)

\begin{abstract}
Macroscopic dark matter (macros) are a broad class of alternative candidates to particle dark matter. These candidates would transfer energy primarily through elastic scattering, and this energy deposition would produce observable signals if a macro were to pass through the atmosphere. We produce constraints for low mass macros from the null observation of bright meteors formed by a passing macro, across two extensive networks of cameras built originally to observe meteorites. The parameter space that could be probed with planned upgrades to the existing array of cameras in one of these networks still currently in use, the Desert Fireball Network in Australia, is estimated.
\end{abstract}

\section{INTRODUCTION}

Assuming General Relativity is the correct theory of gravity on all scales, there is considerable evidence for dark matter. Macroscopic dark matter (macros) is a broad class of dark-matter candidates, with wide stillallowed ranges of masses $M_{x}$ and cross sections $\sigma_{x}$, that represents an alternative to conventional particle dark matter.

Of particular interest would be macros of approximately nuclear density satisfying

$$
\sigma_{x} \approx 2 \times 10^{-10}\left(\frac{M_{x}}{g}\right)^{\frac{2}{3}} \mathrm{~cm}^{2},
$$

as several models for macros describe potential candidates with approximately that density. The idea that macros could be formed entirely within the Standard Model was originally proposed by Edward Witten [1] in the context of a first-order QCD phase transition. Lynn, Nelson and Tetradis 2, and Lynn again [3 subsequently described a more realistic model for StandardModel macros as bound states of nucleons with significant strangeness. Nelson [4 studied the formation of nuggets of strange-baryon matter during a second QCD phase transition - from a kaon-condensate phase to the ordinary phase. Others have considered non-StandardModel versions of such objects and their formation [5]. Although macros satisfying (1) are of particular interest, macros over a wide range of average densities remain possible candidates to explain the open problem of the nature of dark matter.

Colleagues recently determined which regions of macro parameter space remain unprobed [6, 7. A longstanding constraint comes from examination of a slab of ancient mica for tracks that would have been left by the passage of a macro moving at the typical speed of dark matter in the Galaxy. This was used to rule out macros of $M_{x} \leq 55 \mathrm{~g}$ for a wide range of cross sections (see 8 and 9]). Various microlensing experiments have constrained the dark-matter fraction for masses $M_{x} \geq 10^{24} \mathrm{~g}$ [10[13. Reference [14] utilized the full Boltzmann formalism to obtain constraints from macro-photon elastic scattering using the first year release of Planck data. In particular, a sufficiently high dark-matter photon interac- tion will generate distinctive features in the temperature and polarization power spectra at high $\ell$ values. Constraints were derived by comparing the spectra to the latest Planck data, and finding the best-fit cosmological parameters. Prior work had already constrained a similar range of parameter space by showing that the consequence of dark matter interactions with standard model particles is to dampen the primordial matter fluctuations and essentially erase all structures below a given scale (see e.g. [15]). More recently, the existence of massive white dwarfs was used to constrain a significant region of macro parameter space [16. The region of parameter space where macros would have produced a devastating injury similar to a gunshot wound on the carefully monitored population of the western world was also recently constrainted [17.

More work has been done recently to identify additional ways to probe macro parameter space. With colleagues, we have proposed [18] using current Fluorescence Detectors that are designed to study High Energy Cosmic Rays, such as those of the Pierre Auger Observatory 19. Separately, we have suggested [20] that, for appropriate $M_{x}$ and $\sigma_{x}$, the passage of a macro through granite would form long tracks of melted and re-solidified rock that would be distinguishable from the surrounding unmelted granite. A search for such tracks in commercially available granite slabs is planned.

In this manuscript, we describe a way to constrain macro parameter space based on an idea first put forward by Hills [21, who used the non-observation of fastmoving meteors to constrain a wide range of masses of atomic-density dark-matter candidates. We show that far-denser dark-matter candidates were also constrained by this "fireball" null result.

The Desert Fireball Network (DFN) $[22$ is a network of cameras in Australia searching for bright meteors, which are known as bolides. The area covered by the DFN is already much greater than the original network used by Hills, and thus produces more stringent constraints on macros. There are plans for significant DFN upgrades, including increases in observing area, as part of an effort to create a global network. These upgrades will yield still-more-stringent constraints on macros. We estimate below the region of parameter space that could be probed given some reasonable expectations for the eventual size 
and sensitivity of the global network of bolide detectors.

\section{MACRO DETECTABILITY}

In this section, we provide a guide for how we determine constraints from the lack of observation of fastmoving bolides in surveys using in the following section.

A macro transiting the atmosphere will deposit energy along its essentially straight-line path through elastic scattering off molecules in the air. It will do so at a rate

$$
\frac{d E}{d x}=\sigma_{x} \rho_{a t m}(D) v_{x}^{2}
$$

Here $\rho_{a t m}(D) \approx e^{-D / 10 \mathrm{~km}} \mathrm{~kg} \mathrm{~m}^{-3}$ is the density of the atmosphere at altitude $D$, accounting for the atmosphere's scale-height of approximately $10 \mathrm{~km}, \sigma_{x}$ is the geometric cross section of the macro, $v_{x}$ is its speed.

This energy deposition along the macro trajectory will result in some flux of visible light $F\left(\sigma_{x}, v_{x} ; D\right)$ received at the cameras that make up a detector array designed to search for bright meteors. These cameras have a minimum flux $F_{\text {thresh }}$ for a bolide to be visible. Macros that result in a flux greater than the threshold will produce a detectable signal. This implies that at each altitude $D$ there is a minimum velocity $v_{\text {thresh }}\left(\sigma_{x} ; D\right)$ for a macro of cross-section $\sigma_{x}$ to be detectable. In other words, the macro is detectable if $v_{x}>v_{\text {thresh }}\left(\sigma_{x} ; D\right)$ - the smaller $\sigma_{x}$, the larger $v_{\text {thresh }}$ must be (at any given $D$ ).

For definiteness, we assume macros possess a Maxwellian velocity distribution in a frame co-moving with the Galaxy,

$$
f_{M B}\left(v_{x}\right)=\frac{4 \pi v_{x}^{2}}{\left(\pi v_{v i r}^{2}\right)^{3 / 2}} e^{-\left(\frac{v_{x}}{v_{v i r}}\right)^{2}}
$$

where $v_{v i r} \approx 250 \mathrm{~km} \mathrm{~s}^{-1}\left[23\right.$, but cut off at $v_{x, \text { esc }} \sim$ $550 \mathrm{~km} \mathrm{~s}^{-1}$ - the escape velocity from the Galaxy at the Sun's position. The macro flux detectable from altitude $D$ is given by the cumulative velocity distribution function - specifically the integral of the product of the relative velocity between the macro and the Earth and the macro velocity-distribution function (transformed to the rest frame of the solar system $f_{M B S S}\left(v_{x}\right)$ from $v_{\text {thresh }}\left(\sigma_{x} ; D\right)$ to $v_{x, e s c}$.

The local mass density of dark matter is fixed by Galactic dynamics. For simplicity of interpretation, we consider all macros to be of a single mass and size, even though a broad mass distribution is a reasonable possibility in the context of a composite dark-matter candidate. The macro flux is therefore inversely proportional to the macro mass, and we must have

$$
\Gamma_{\min } \leq \Gamma\left(M_{x}, \sigma_{x}\right) \equiv A_{d e t} \frac{\rho_{D M}}{M_{x}} \int_{v_{\text {thresh }}\left(\sigma_{x} ; D\right)}^{v_{e s c}} v_{x} f_{M B S S}\left(v_{x}\right) d v_{x}
$$

where $\rho_{D M} \simeq 5 \times 10^{-25} \mathrm{~g} \mathrm{~cm}^{-3}$ [24], and $A_{\text {det }}$ is the projected area on the sky covered by the network of cameras in the bolide survey. Consequently, the smaller $v_{x, t h r e s h}$, the lower $\sigma_{x}$ we can probe at the cost of only being sensitive to a fraction of the macros in the Maxwellian distribution, corresponding to a maximum macro mass. The smaller $\sigma_{x}$ is, the higher $v_{x, \text { thresh }}$ needs to be to produce enough photons for an event to be detectable. That higher $v_{x, \text { thresh }}$ means that a smaller fraction of the macros are detectable, so to have a high enough event rate, the overall number density of macros must be higher, i.e. they must be of lower mass. Higher $\sigma_{x}$ thus means lower maximum probable mass.

Equation (4) allows us to determine, as a function of $\sigma_{x}$, the maximum mass $M_{x}$ that we can probe, so long as we know the velocity distribution as a function of altitude. The speed of a macro traveling through the atmosphere is expected to evolve as

$$
v(x)=v_{0} e^{-\langle\rho \Delta\rangle \frac{\sigma_{x}}{M_{x}}} .
$$

Here $\langle\rho \Delta\rangle$ is the integrated column density traversed along the trajectory from the point of impact to the lo- cation $\mathrm{x}$,

$$
\langle\rho \Delta\rangle \equiv \int_{l} e^{-D(x) / 10 \mathrm{~km}} \mathrm{~kg} \mathrm{~m}{ }^{-3} d x,
$$

where $l$ represents the trajectory of the macro and $10 \mathrm{~km}$ in the exponent is the atmospheric scale height.

For large enough values of $\sigma_{x} / M_{x}$, macros will not traverse the Earth and only macros at high altitude will produce visible signals. This could be allowed to determine the maximum value of $\sigma_{x} / M_{x}$ accessible by the bolide network. However, in order to be detected by the camera network, a macro must remain intact as it passes through the atmosphere. Ordinary meteors often break up.

We expect sufficiently dense objects to be stable enough to survive passage through the upper atmosphere. The precise threshold density depends on the microscopic physics of the macro, however we can get a sense of how dense by imagining the macro is made of baryons, and that the logarithm of the binding energy per baryon $E_{b}$ scales linearly with the logarithm of the density between atomic density $\left(\rho_{\text {atomic }} \simeq 1 \mathrm{~g} / \mathrm{cm}^{-3}, E_{b} \simeq 10 \mathrm{eV}\right)$ and nuclear density $\left(\rho_{\text {nuclear }} \simeq 10^{14} \mathrm{~g} / \mathrm{cm}^{-3}, E_{b} \simeq 1 \mathrm{MeV}\right)$. 
This yields an expression for the scaling between binding energy and density

$$
E_{b} \sim 10 e V\left(\frac{\rho_{x}}{g / m^{-3}}\right)^{\frac{3}{7}}
$$

where

$$
\rho_{x}=\frac{3 M_{x} \pi^{\frac{1}{2}}}{4 \sigma_{x}^{\frac{3}{2}}} .
$$

We require the energy transferred be less than the binding energy per baryon multiplied by the number of baryons in the macro

$$
E_{b} \frac{M_{x}}{m_{b}} \geq \rho \sigma_{x} v_{x}^{2} L .
$$

Considering just the densest part of the atmosphere, i.e. that closest to the ground, i.e. $\rho \sim 10^{-4} \mathrm{~g} \mathrm{~cm}^{-3}$ in the scale length closest to the ground, we find the above expression translates into a bound

$$
\frac{\sigma_{x}}{\mathrm{~cm}^{2}} \lesssim 4 \times 10^{-3}\left(\frac{M_{x}}{\mathrm{~g}}\right)^{\frac{20}{23}}
$$

This bound is likely too stringent in that most macros at these large cross sections will be detectable at higher altitudes with significantly lower atmospheric densities. However, we use this as our upper bound in Figure 1.

\section{DETECTION THRESHOLDS}

A bolide refers to a very bright meteorite with $M_{v} \geq$ -5 . The original bolide networks operating in the 1960s, '70s and ' 80 s were reliably capable of detecting objects of absolute visual magnitude $M_{v} \leq-5$ [25]. The absolute magnitude [26] of a bolide is related to its luminosity by

$$
M_{v}=6.8-2.5 \log _{10}\left(\mathcal{L} / \mathcal{L}_{0}\right)
$$

where $\mathcal{L}$ is the visible luminosity of a regular meteor and $\mathcal{L}_{0}=1 W$. Based on the criterion $M_{v} \leq-5$, bolides would have been detectable when their luminosity in the visual spectrum exceeded $\sim 50000 \mathrm{~W}$. Bolides are typically seen at altitudes $\sim 100 \mathrm{~km}$. This implies a flux received at ground level of $F_{\text {thresh }} \geq 10^{-8} \mathrm{~W} \mathrm{~m}^{-2}$.

The DFN reported a limiting apparent magnitude $m_{V}=0.5$ 22]. This is an instrument-specific threshold for the faintest object that is reliably detectable. We infer a minimum flux that must be received at the DFN camera of $F_{\text {thresh }} \gtrsim 10^{-8} \mathrm{~W} \mathrm{~m}^{-2}$, similar to the value derived above.

A meteor (including a macro transiting the atmosphere) must exceed this threshold brightness to be detectable by the network cameras. This brightness depends on both the intrinsic luminosity of the meteor and its distance from the cameras. We neglect the effect of photon scattering along the path from the macro trajectory to the camera, which is a small correction [27. The actual flux received is therefore proportional to the intrinsic luminosity of the macro as it passes through the atmosphere (which depends on $v_{x}$ and $\sigma_{x}$ for the macro), and inversely proportional to the square of the distance from the macro trajectory to the camera. As macros are expected to be significantly denser than an ordinary meteorite, they are unlikely to fragment at high altitude like ordinary meteorities. Thus they are expected to be observable much closer to the ground.

We treat the entire emission region produced by the macro as a point source within the field-of-view (FOV) of a single camera pixel. This is reasonable given that the pixels have a small FOV 22] of $\left(0.036^{\circ}\right)^{2}$, i.e. the transverse length of the path seen by a pixel is significantly less than the distance between the macro and the pixel.

As a macro at altitude $D$ passes through the angle $\theta$ subtended by the FOV of a pixel, it traverses a distance $L=D \theta$ - depositing energy $L d E / d x$ (per equation (2)), over a time $L / v(x)$. This creates a plasma, which persists for a time $t_{I 0}$ [27]. As the plasma cools it emits a fraction $\epsilon$ of its energy into the part of the (visible) spectrum to which the camera is sensitive. The flux incident on the camera pixel is then

$$
\begin{aligned}
F & =\min \left(\frac{v(x)}{L}, \frac{1}{t_{I 0}}\right) \epsilon \frac{d E}{d x} \frac{L}{4 \pi D^{2}} \\
& =3 J m^{-2} \min \left(\frac{v(x)}{L}, \frac{1}{t_{I 0}}\right)\left(\frac{\sigma_{x}}{c m^{2}}\right)^{2}\left(\frac{v(x)}{250 k m s^{-1}}\right)^{4} e^{-\frac{3 D}{20 k m}} \frac{k m}{D} .
\end{aligned}
$$

For macros, we have defined an analogous quantity $\mathcal{L}$ as

$$
\mathcal{L}=\min \left(\frac{v(x)}{L}, \frac{1}{t_{I 0}}\right) \epsilon \frac{d E}{d x} L
$$

where $\epsilon$ is the fraction of energy that emerges into visible wavelengths previously calculated in [18 for macros 
passing through the atmosphere,

$$
\begin{aligned}
\epsilon & =\frac{\mathrm{N}_{\gamma}^{\operatorname{thin}} \bar{E}}{\frac{1}{2} \rho v_{x}^{2} \sigma_{x} \mathrm{~L}} \\
& \approx 2 \times 10^{2}\left(\frac{\sigma_{x}}{c m^{2}}\right)^{2}\left(\frac{v(x)}{250 k m s^{-1}}\right)^{4} e^{-\frac{3 D}{10 k m}}
\end{aligned}
$$

where $\mathrm{N}_{\gamma}^{\text {thin }}$ is the number of photons emitted by the plasma, and $\bar{E}$ is the average energy of those photons. We expect that at large cross-sections $\sigma_{x} \gtrsim 2 \times 10^{-3} \mathrm{~cm}^{2}$, $\epsilon$ would eventually saturate at some fraction of the total energy deposited. (See section 2 of 27] for a discussion on why this is the case.) We have divided by the larger of the two timescales present in the problem, $\frac{L}{v_{X}}$, which is the pixel crossing time, and $t_{I 0}$, which is the time of existence of the plasma produced by the macro. The larger of the two timescales is what determines the flux produced at the camera.

Using (12) and (14), taking account of the distribution in altitude of macro trajectories, and using the macro velocity distribution, we can ascertain the detectability of macros of particular $\sigma_{x}$, by requiring $F \geq F_{\text {thresh }}$ sometime in their passage through the atmosphere.

To constrain $\sigma_{x}$ as a function of $M_{x}$, we enforce the null observation of a fast-moving macro that would have produced a detectable bolide. For macros large enough to be detected, the expected number of events that a survey should have seen is determined by multiplying (4) by the relevant observation time of the survey. We obtain $v_{\text {thresh }}$ from 12 and the requirement that $F \geq F_{\text {thresh }}$, taking care to note that $v_{\text {thresh }}$ is its value at the top of the atmosphere, not at altitude $D$. Of course, $v_{\text {thresh }}$ depends on $D$ and so we must sample altitudes appropriately, as described below.

The passage of a macro through the field of view of a survey is a Poisson process. The probability $P(n)$ of $n$ passages over a given exposure time, follows the distribution:

$$
P(n)=\frac{N_{\text {events }}{ }^{n}}{n !} e^{-N_{\text {events }}}
$$

where $N_{\text {events }}$ is, as computed above, the expected number of events per interval, and varies by detector area and exposure time.

If a macro were to cross the field of view of one of these networks, the network would observe a bolide moving far too fast to be bound within the Solar System. This presents an easy way to distinguish between bolides formed from solar-system meteoroids and macros. 28. Thus, the non-observation of such a fast-moving bolide allows us to constrain macros that are big enough to have produced a detectable signal. Requiring $N_{\text {events }} \geq 3$ (obtained by setting the probability $P(n=0) \leq 0.05$ in (15), gives us a $95 \%$ C.L. contraint that macros of at most the selected mass and at least the selected crosssection do not constitute all the dark matter.

\section{Analysis}

To determine the regions of parameter space that are constrained or could be further probed we proceed as follows:

- Iterate $v_{x}$ over the range of allowed values $(0 \mathrm{~km} / \mathrm{s}$ $550 \mathrm{~km} / \mathrm{s})$.

- For each value of $v_{x}$, iterate over a wide range of altitudes $10 \mathrm{~m} \leq D \leq 100 \mathrm{~km}$. The lower limit comes from the dependence of the event rate on $D$. Taking each camera to have a roughly conical field-of-view, and noting that the cameras are $\sim 100 \mathrm{~km}$ apart, $A_{\text {det }}$ will scale as $D^{2}$. For $D \leq 10 \mathrm{~m}$, the event rate is too low for any unconstrained parameter space to be probed.

The upper bound comes from the strong dependence of the incident flux $F$ on $D$, as seen in $\sqrt{12}$. For $D \gtrsim 100 \mathrm{~km}$, we find that the macro crosssection necessary to produce a detectable signal is already ruled out by other considerations.

- For each value of $D$, determine the smallest and largest cross-sections that can be probed from (12) (requiring that $F \geq F_{\text {thresh }}$ ), and the maximum mass that may be probed from (4) (requiring that $N_{\text {events }} \geq 3$ ). The upper bound on the range of $\sigma$ that can be probed, is always determined by by requiring macros be sufficiently dense to survive passage through the atmosphere, which we conservatively take to be $10^{3}$ times atomic density.

- From the above iterations, for each value of $M_{x}$ determine the range of values of $\sigma_{x}$ that has been constrained or can be probed.

These final values of $M_{x}$ and $\sigma_{x}$ are presented in Figure 1.

\section{CONSTRAINTS FROM PAST METEORITE NETWORKS}

We derive constraints on macros from a lack of visible evidence of them transiting through the atmosphere across the fields of view of two meteorite networks: a combination of the U.S. Prairie Network, the Canadian Network, and the Eastern European Network, which we refer to collectively as the PCE network, which operated in the $60 \mathrm{~s}, 70 \mathrm{~s}$ and $80 \mathrm{~s}$; 21] and the the Desert Fireball Network, 22. currently operating in Australia.

\section{PCE network}

No fireballs moving fast enough to have an origin beyond the solar system were observed in a large-area survey over an "effective" (i.e. scaled to the full area of the Earth) period of 30 hours [21, so $A_{\text {det }} t \sim A_{\oplus} 30$ hours. 
Using 12 and 14, and requiring $F \geq F_{\text {thresh }}=$ $10^{-8} \mathrm{~W} \mathrm{~m} \mathrm{~m}^{-2}$, macro visibility by PCE network cameras requires

$$
\sigma_{x} \geq 2 \times 10^{-4} \mathrm{~cm}^{2}\left(\frac{250 \mathrm{~km} / \mathrm{s}}{v_{x}}\right)^{2}\left(\frac{D}{\mathrm{~km}}\right)^{1 / 2} e^{3 D / 20 \mathrm{~km}}
$$

In general, the plasma lifetime $t_{I 0}$ is larger than the pixel crossing time of the macro, and is the term relevant in 12. Thus

$$
N_{\text {events }}=1.9 \times 10^{6} f_{x} f_{\text {det }}\left(\sigma_{x}\right) \frac{g}{M_{x}},
$$

where $f_{x} \equiv \Omega_{x} / \Omega_{D M}$ is the fraction of the dark matter in macros. (We continue to assume that all macros have a single mass $M_{x}$ and cross-section $\sigma_{x}$.)

The non-observation of any fast-moving fireballs, allows us to conclude, at the 95 per cent confidence level, that

$$
f_{x} \leq \frac{M_{x}}{6 \times 10^{5} g} \frac{1}{f_{\text {det }}\left(\sigma_{x}\right)}
$$

for macros satisfying $(16)$. These results are presented in Figure 1 in green with black diagonal hatching.

\section{Desert Fireball Network}

In this section we recalculate, using the framework described above, the region of parameter space excluded by the non-observation of an extra solar bolide by the Desert Fireball Network (DFN). DFN is an extensive array of cameras monitoring approximately one-third of Australian skies for bolides with a minimum magnitude of $m_{\nu} \approx 0.5[22$.

The Australian Nullarbor plain is a good site for a fireball camera network due to ideal viewing conditions. The lack of vegetation and pale geology make recovery of a fallen meteor easier. These reasons, along with the relative ease of setting up an extensive network of cameras at a reasonable price 22 led to creation of the DFN over the last decade, and its current state of more than 50 cameras observing most nights of the year [29]. This large area will allow new constraints to be produced relative to the previous network of cameras.

\section{Current Constraints}

The expression for the minimum cross- section that can be probed as a function of $D$ and $v_{x}$ remains unchanged from 16, as the specifics of the cameras in the DFN and PCE aren't significantly different, as suggested by the equlaity of the two threshold fluxes reported above.

The DFN array has been running with an active detection area of $A_{d e t} \approx 2 \times 10^{6} \mathrm{~km}^{2}$ for almost three years
22. Thus the expected number of fireball events that the survey should have seen is

$$
N_{\text {events }}=1.2 \times 10^{7} f_{x} f_{\text {det }}\left(\sigma_{x}\right) \frac{g}{M_{x}} .
$$

We conclude that

$$
f_{x} \leq \frac{M_{x}}{4 \times 10^{6} g} \frac{1}{f_{\text {det }}\left(\sigma_{x}\right)}
$$

for macros satisfying (16). These constraints are presented in Figure 1 in green with no hatching.

\section{Future parameter space that may be probed}

There are concrete plans to form a global array of cameras to increase the observing area for meteors [22]. The current array is observing approximately $0.5 \%$ of the Earth's surface area.

Since an important objective of such meteormonitoring programs is to recover the remnant meteorite, they are most likely to be extended over desert regions, which also are the places most likely to provide the highest fraction of times with good viewing conditions. Deserts comprise approximately $10 \%$ of the Earth's surface, which is therefore an optimistic upper limit to the coverage of future arrays. Realistically, the planned duration of a meteor search is unlikely to be longer than 30 years, a factor of 3 longer than the current age of the DFN. We can thus expect at most a factor of 60 increase in total exposure.

This yields

$$
N_{\text {events }}=1.2 \times 10^{9} f_{x} f_{\text {det }}\left(\sigma_{x}\right) \frac{g}{M_{x}} .
$$

The continued non-observation of fast-moving fireballs during an observing time 3 times the current value by an array 20 times larger than the current size would place the constraint

$$
f_{x} \leq \frac{M_{x}}{4 \times 10^{8} g} \frac{1}{f_{\text {det }}\left(\sigma_{x}\right)}
$$

for macros satisfying (16). This region is presented in green hatching in Figure 1. In Figure 1, we have also presented projections from a recent study [27] of the Fluorescence Detectors (FDs) that are components of large ultra-high-energy cosmic-ray detector arrays, for comparison to the results presented in this manuscript. While the methods outlined here will allow us to probe higher masses, the FDs will allow a greater sensitivity to smaller cross-sections. Thus, a complimentary approach will be ideal for probing as much of the parameter space as possible.

The actual value of $M_{x}$ that will eventually be probed by the expanded DFN network will of course depend on the final size of the network of cameras and its total live time. Values as high as $M_{x} \sim 10^{9}$ g could be probed. 


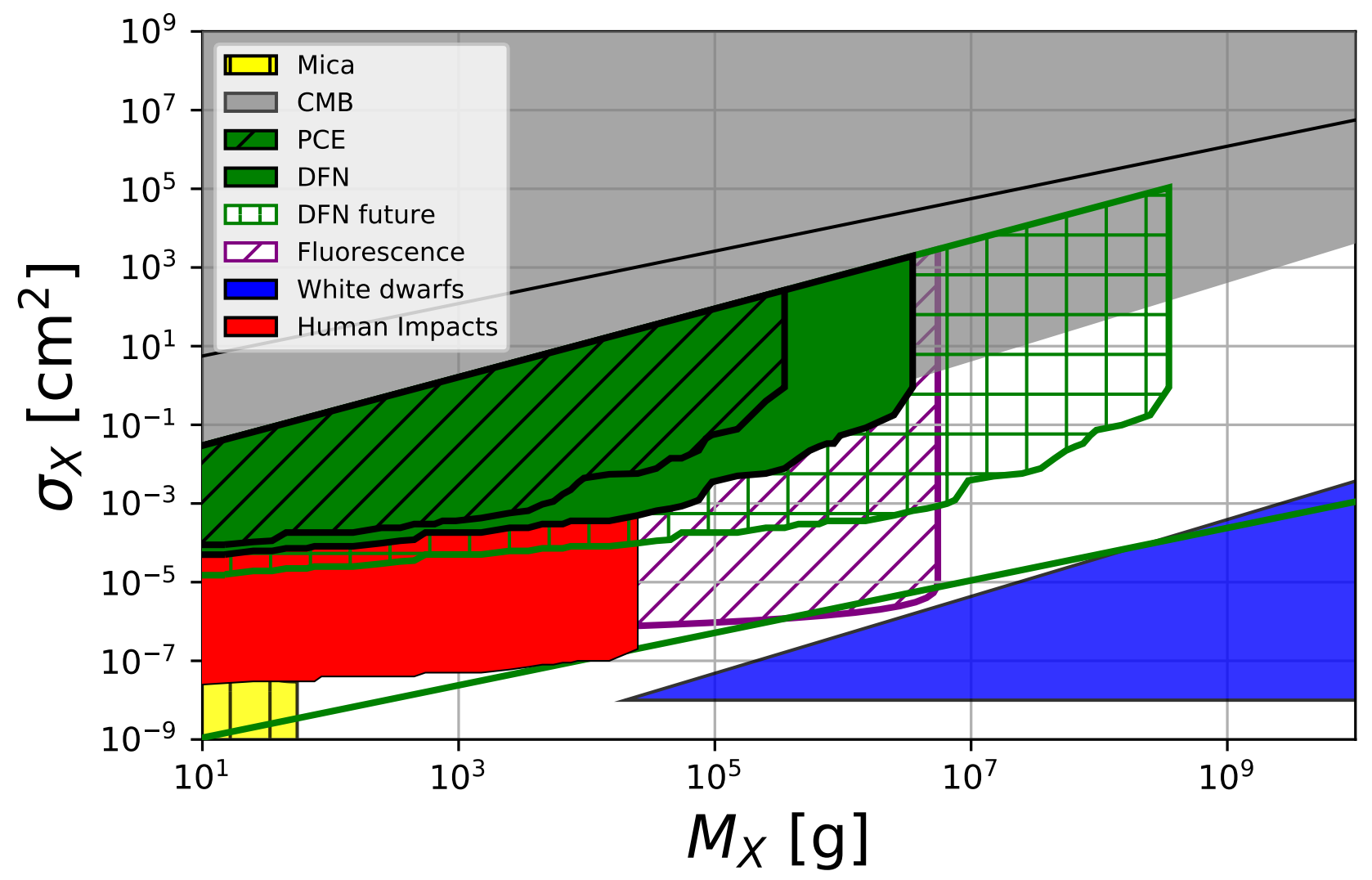

Figure 1. Constraints (solid green) derived from the null observation of bolides produced by a passing macro in the combination of the U.S. Prairie, Canadian, and Eastern European (PCE) bolide networks that existed in the 1960's - 1980's, and the currently operational Desert Fireball Network (DFN), and the region of parameter space that may eventually be probed given certain reasonable assumptions about the final size of the DFN network (green hatching). The details of the green regions are explained in the text. The region currently excluded by examination of ancient mica 8, 9] is shown in yellow with vertical hatching; the grey region is excluded from the effects of CMB photons scattering off the macros [14; the blue region from the continued existence of white dwarfs [16; and the red from the lack of human impacts [17. The purple (hatched) region is accessible in the future to the Fluorescence Detectors that are components of certain Ultra-High-Energy Cosmic Ray detectors e.g., those that may result from the JEM-EUSO program [27].

\section{CONCLUSION}

We have produced constraints from the nonobservation of "extrasolar meteors," i.e. bolides produced by the passage of a macro with a sufficiently large geometric cross section $\sigma_{x}$. We have identified a region of macro parameter space that could potentially be probed by expansion of the DFN network of meteorite cameras over the coming years. This region represent a significant (up to 60x) improvement on the mass-reach of current constraints.

Since it is unclear what part of the available parameter space macros should occupy, it is vital to explore as much of it as possible.

The idea outlined here is similar to the use of Fluorescence Detectors to look for photons produced by a macro passing in the vicinity [18. The minimum values of $\sigma_{x}$ that could be probed by planned or potential expansions of the DFN are not competitive with the lowest values of $\sigma_{x}$ that could be probed using FDs [18]. However, it is very likely easier to reach higher masses sooner with an upgraded bolide network than with an FD, because of the relative (!) ease of expanding the existing bolide networks 22 compared to building an appropriately configured FD network. The FD network is needed to probe to lower $\sigma_{x}$, especially to reach the potentially most interesting nuclear densities.

An interesting corollary to this estimate of the future parameter space that could be probed by the DFN, is that it seems unlikely that macro masses beyond $\sim 10^{9} \mathrm{~g}$ could be probed by any purpose-built terrestrial detector assuming even an observation time of a century and a target area the size of the Earth. Terrestrial probes (eg. ancient rocks [8, 9, 20]) could have been continuously exposed for up to $3 \times 10^{9}$ years, but we are unlikely 
to carefully examine the more than $1 \mathrm{~km}^{2}$ that would be needed to push beyond $M_{x}=10^{9} \mathrm{~g}$. It will therefore require innovative thinking about astrophysical probes (eg. [16] ) to probe the very highest possible macro masses.

\section{ACKNOWLEDGMENTS}

This work was partially supported by Department of Energy grant de-sc0009946 to the particle astrophysics theory group at CWRU.
[1] E. Witten, Physical Review D 30, 272 (1984)

[2] B. W. Lynn, A. E. Nelson, and N. Tetradis, Nuclear Physics B 345, 186 (1990).

[3] B. W. Lynn, "Liquid phases in SU(3) Chiral Perturbation Theory: Drops of Strange Chiral Nucleon Liquid and Ordinary Chiral Heavy Nuclear Liquid," (2010), arXiv:1005.2124.

[4] A. E. Nelson, Physical Letters B240, 179 (1990)

[5] A. R. Zhitnitsky, Journal of Cosmology and Astroparticle Physics 2003, 010 (2003)

[6] D. M. Jacobs, G. D. Starkman, and B. W. Lynn, (2014), 10.1093/mnras/stv774, arXiv:1410.2236.

[7] D. M. Jacobs, A. Weltman, and G. D. Starkman, Physical Review D 91, 115023 (2015)

[8] P. B. Price, Physical Review D 38, 3813 (1988)

[9] A. De Rujula and S. L. Glashow, Nature 312, 734 (1984)

[10] C. Alcock et al., The Astrophysical Journal 550, L169 (2001)

[11] K. Griest, A. M. Cieplak, and M. J. Lehner, Physical Review Letters 111, 181302 (2013).

[12] P. Tisserand et al., Astronomy \& Astrophysics 469, 387 (2007)

[13] B. J. Carr, K. Kohri, Y. Sendouda, and J. Yokoyama, Physical Review D 81, 104019 (2010)

[14] R. J. Wilkinson, J. Lesgourgues, and C. Bøhm, Journal of Cosmology and Astroparticle Physics 2014, 026 (2014)

[15] C. Bøehm, P. Fayet, and R. Schaeffer, Physics Letters B 518, 8 (2001)

[16] P. W. Graham, R. Janish, V. Narayan, S. Rajendran, and P. Riggins, Physical Review D 98, 115027 (2018).

[17] J. S. Sidhu, R. J. Scherrer, and G. Starkman, "Death by dark matter," (2019), arXiv:1907.06674.

[18] J. S. Sidhu, R. M. Abraham, C. Covault, and G. Starkman, (2018), 10.1088/1475-7516/2019/02/037, arXiv:1808.06978.

[19] J. Abraham et al., Nuclear Instruments and Methods in Physics Research Section A: Accelerators, Spectrometers, Detectors and Associated Equipment 620, 227 (2010).

[20] J. S. Sidhu, G. Starkman, and R. Harvey, Physical Review D 100 (2019), 10.1103/physrevd.100.103015.

[21] J. G. Hills, The Astronomical Journal 92, 595 (1986).

[22] R. M. Howie, J. Paxman, P. A. Bland, M. C. Towner, M. Cupak, E. K. Sansom, and H. A. R. Devillepoix, Experimental Astronomy 43, 237 (2017).

[23] This is the distribution of macro velocities in a nonorbiting frame moving with the Galaxy. When considering the velocity of macros impacting the atmosphere, (3) is modified by the motion of the Sun and Earth in that frame, and by the Sun's and Earth's gravitational potential. We have taken into account these effects (as explained, for example, in 30), except the negligible effect of Earth's gravitational potential.
[24] J. Bovy and S. Tremaine, The Astrophysical Journal 756, $89(2012)$

25] R. E. Mccrosky and J. H. Boeschenstein, Optical Engineering 3 (1965), 10.1117/12.7971304.

[26] E. J. Opik, Quarterly Journal of the Royal Meteorological Society 85, 320 (1959).

[27] J. S. Sidhu, R. M. Abraham, C. Covault, and G. Starkman, JCAP 1902, 037 (2019), arXiv:1808.06978 [astroph.HE]

[28] Interstellar meteors could provide false positives for macros. In 30 years of CNEOS data, it has been determined that at most 1 such interstellar meteor has been observed [31, with even this conclusion being uncertain 32. However, CNEOS is currently sensitive to objects that are $\gtrsim 140 \mathrm{~m}$ across, and so extrapolating abundances to the much smaller objects we are considering is uncertain. We take the background rate to be zero, but acknowledge that the detection of a fast-moving bolide would require follow up investigation to distinguish between a meteor and a macro.

[29] "The research - fireballs in the sky," http:// fireballsinthesky.com.au/the-research/, accessed: 2019-06-13.

[30] K. Freese, M. Lisanti, and C. Savage, Reviews of Modern Physics 85, 1561 (2013).

[31] A. Siraj and A. Loeb, "Discovery of a meteor of interstellar origin," (2019), arXiv:1904.07224

[32] L. Billings, "Did a meteor from another star strike earth in 2014?" https://www.scientificamerican.com/article/did-ameteor-from-another-star-strike-earth-in-2014/ (2019), Accessed: October 23rd 2019. 